\title{
Some Iterative Numerical Methods for a Kind of System of Mixed Nonlinear Variational Inequalities
}

\author{
Shuilian Xie ${ }^{1}$, Hongru $\mathrm{Xu}^{1}$ \& Huiqing Huang ${ }^{1}$ \\ ${ }^{1}$ School of Mathematics, Jiaying University, Meizhou, Guangdong, P. R. China \\ Correspondence: Hongru Xu, School of Mathematics, Jiaying University, Meizhou, Guangdong 514015, P. R. \\ China. E-mail: hrxu001@163.com
}

Received: October 8, 2013 Accepted: November 26, 2013 Online Published: January 9, 2014

doi:10.5539/jmr.v6n1p65 URL: http://dx.doi.org/10.5539/jmr.v6n1p65

\begin{abstract}
In this paper, we study the numerical solution of a type of system of mixed nonlinear variational inequalities in a Banach space. Using the properties of $\eta$-proximal mapping, we construct some iterative algorithms for solving systems of mixed nonlinear variational inequalities. Moreover, we establish the convergence theorems for the proposed numerical methods.
\end{abstract}

Keywords: system of mixed nonlinear variational inequalities, $\eta$-proximal mapping, iterative algorithm, convergence

\section{Introduction}

Variational inequality is a kind of very important nonlinear problems and a powerful tool for studying a wide class of problems arising in engineering, physics, economics, optimal control and so on, see for example Duvaut and Lions (1976), Facchinei and Pang (2003), and references therein. During the last decades, researchers have made great progress in obtaining numerical solutions of variational inequality, such as projection method and its variations, linear approximation method, smoothing Newton method, domain decomposition method, etc. Since standard projection methods depend on the inner product on Hilbert spaces, this kind of technique cannot be extended to variational inequality in Banach spaces. Even more, for mixed variational inequalities, projection method is neither appropriate, since it is difficult to find the projection.

Recently, thanks to emergence of new kinds of numerical solutions, variational inequalities have been extended in many directions. One of the most important extensions is the system of variational inequalities, see Ferris and Pang (1997), Kazmi and Khan (2007), Verma (2007), and references therein. Huang and Noor (2007) discussed the convergence of projection method for a kind of system of variational inequalities in Hilbert spaces. Verma (2001) studied the numerical solution for a kind of a system of nonlinear variational inequalities in Hilbert space, and proposed a series of projection methods. In this paper, we will go on doing the work in this area. We study the numerical solution of system of mixed nonlinear variational inequalities in Banach space. We introduce the definition of $\eta$-proximal mapping for a proper subdifferentiable functional. Using the properties of $\eta$-proximal mapping, we construct some iterative algorithms for solving systems of mixed nonlinear variational inequalities. Morever, we establish the convergence theorems for the proposed numerical methods.

\section{Preliminaries}

First we give a hypothesis which will be used throughout the paper.

Hypothesis A Let $B$ be a reflexive Banach space, $B^{*}$ be the dual space $B$ and $\langle\cdot, \cdot\rangle$ denote the pairing between $B^{*}$ and $B$. Let $\phi: B \rightarrow(-\infty,+\infty]$ be a proper lower semicontinuous and subdifferentiable functional.

Let $2^{B^{*}}$ denote all subsets of $B^{*}$. Let $T: B \rightarrow B^{*}$ be single-valued mappings. We consider the following system of mixed nonlinear variational inequalities (denoted by SMNVI): Find $\left(x^{*}, y^{*}\right) \in B \times B$, such that

$$
\begin{array}{ll}
\left\langle\gamma_{1} T\left(y^{*}\right)+x^{*}-y^{*}, x-x^{*}\right\rangle \geq \phi\left(x^{*}\right)-\phi(x), & \gamma_{1}>0, \quad \forall x \in B, \\
\left\langle\gamma_{2} T\left(x^{*}\right)+y^{*}-x^{*}, y-y^{*}\right\rangle \geq \phi\left(y^{*}\right)-\phi(y), & \gamma_{2}>0, \quad \forall y \in B .
\end{array}
$$

We first recall the following definitions and some known results. 
Definition 1 The following mapping $J: B \rightarrow 2^{B^{*}}$ is said to be a normal dual mapping:

$$
J(x)=\left\{f \in B^{*}:\langle f, x\rangle=\|f\| \cdot\|x\|,\|f\|=\|x\|\right\}, \quad \forall x \in B .
$$

Definition 2 Let Hypothesis A hold and $\eta: B \rightarrow B^{*}$ be a mapping. Mapping $x^{*} \mapsto x$, denoted by $x=J_{\rho}^{\phi}\left(x^{*}\right)$, is said to be a $\eta$-proximal mapping for $\phi$, if for any $x^{*} \in B^{*}$ and constant $\rho>0$, there exists $x \in B$ satisfying

$$
\left\langle\eta x-x^{*}, y-x\right\rangle+\rho \phi(y)-\rho \phi(x) \geq 0, \quad \forall y \in B .
$$

Definition 3 Let $A: B \rightarrow B^{*}$ be a single mapping. $A$ is said to be $\alpha$-strongly monotone, if for any $x, y \in B$, there exists constant $\alpha>0$, such that

$$
\langle A x-A y, x-y\rangle \geq \alpha\|x-y\|^{2} .
$$

Hypothesis B Let $\eta: B \rightarrow B^{*}$ be an $\alpha$-strongly continuous mapping.

Definition 4 Let $B$ be a Banach space, $T: B \rightarrow B$ is said to be $s$-Lipschitz continuous, if there exists $s>0$ such that

$$
\|T(x)-T(y)\| \leq s\|x-y\|, \quad \forall x, y \in B .
$$

By the definition of subdifferentiable and (2), we have $x^{*}-\eta x \in \rho \partial \phi(x)$, hence

$$
x=J_{\rho}^{\phi}\left(x^{*}\right)=(\eta+\rho \partial \phi)^{-1}\left(x^{*}\right) .
$$

Remark 1 If $B=H$ is a Hilbert space, $\eta: H \rightarrow H$ is an identity mapping on $H$, and $\phi$ is a proper convex subdifferentiable functional, then $\eta$-proximal mapping for $\phi$ degenerates to a resolvent operator on $H$.

Lemma 1 (Xia \& Huang, 2008) Let Hypothesises $A$ and $B$ hold. Then for any $x^{*} \in B^{*}$ and any $\rho>0$, there exists unique $x \in B$ such that

$$
\left\langle\eta x-x^{*}, y-x\right\rangle+\rho \phi(y)-\rho \phi(x) \geq 0, \quad \forall y \in B .
$$

That is, $x=J_{\rho}^{\phi}\left(x^{*}\right), \eta$-proximal mapping for $\phi$ is well defined.

Lemma 2 (Xia \& Huang, 2008) Let Hypothesises $A$ and $B$ hold. Then $\eta$-proximal mapping $J_{\rho}^{\phi}=(\eta+\rho \partial \phi)^{-1}$ is $\frac{1}{\alpha}$-Lipschitz continuous. If the subdifferentiable $\partial \phi: B \rightarrow 2^{B^{*}}$ of $\phi$ is $\xi$-strongly monotone, then $\eta$-proximal mapping $J_{\rho}^{\phi}=(\eta+\rho \partial \phi)^{-1}$ is $\frac{1}{\alpha+\rho \xi}$ Lipschitz continuous.

Lemma 3 Let Hypothesises $A$ and $B$ hold. Then, $\left(x^{*}, y^{*}\right) \in B \times B$ is the solution of $(1)$ if and only if

$$
\begin{aligned}
& x^{*}=J_{\rho}^{\phi}\left[\eta\left(x^{*}\right)-\rho\left(\gamma_{1} T\left(y^{*}\right)+x^{*}-y^{*}\right)\right], \\
& y^{*}=J_{\rho}^{\phi}\left[\eta\left(y^{*}\right)-\rho\left(\gamma_{2} T\left(x^{*}\right)+y^{*}-x^{*}\right)\right],
\end{aligned}
$$

where $J_{\rho}^{\phi}=(\eta+\rho \partial \phi)^{-1}, \rho>0$ is a constant.

Proof. Let $\left(x^{*}, y^{*}\right)$ satisfy (3). Since $J_{\rho}^{\phi}=(\eta+\rho \partial \phi)^{-1}$, we have (3) holds if and only if $\left(x^{*}, y^{*}\right)$ satisfies

$$
\begin{aligned}
& \eta\left(x^{*}\right)-\rho\left(\gamma_{1} T\left(y^{*}\right)+x^{*}-y^{*}\right) \in \eta\left(x^{*}\right)+\rho \partial \phi\left(x^{*}\right), \\
& \eta\left(y^{*}\right)-\rho\left(\gamma_{2} T\left(x^{*}\right)+y^{*}-x^{*}\right) \in \eta\left(y^{*}\right)+\rho \partial \phi\left(y^{*}\right) .
\end{aligned}
$$

By the definition of functional subdifferentiable, (4) is equivalent to

$$
\begin{aligned}
& \left\langle\gamma_{1} T\left(y^{*}\right)+x^{*}-y^{*}, x-x^{*}\right\rangle \geq \phi\left(x^{*}\right)-\phi(x), \quad \forall x \in B, \\
& \left\langle\gamma_{2} T\left(x^{*}\right)+y^{*}-x^{*}, y-y^{*}\right\rangle \geq \phi\left(y^{*}\right)-\phi(y), \quad \forall y \in B .
\end{aligned}
$$

In summary, $\left(x^{*}, y^{*}\right)$ is the solution of (1) if and only if $\left(x^{*}, y^{*}\right)$ satisfies (3), which completes the proof.

Lemma 4 (Verma, 2001) Let $\left\{\delta_{n}\right\}_{n=0}^{\infty}$ be a non negative sequence, and satisfy the following inequality

$$
\delta_{n+1} \leq\left(1-\lambda_{n}\right) \delta_{n}+\sigma_{n}, \quad \forall n \geq 0,
$$

where, $\lambda_{n} \in[0,1], \sum_{n=0}^{\infty} \lambda_{n}=\infty$, and $\sigma_{n}=o\left(\lambda_{n}\right)$, then $\lim _{n \rightarrow \infty} \delta_{n}=0$. 


\section{Numerical Methods and Convergence}

Based on Lemma 3, we propose the following iterative numerical methods for (1).

Method 1 Let Hypothesises A and B hold. For any $\left(x_{0}, y_{0}\right) \in B \times B$, calculate $\left(x_{n+1}, y_{n+1}\right) \in B \times B$ :

$$
\begin{aligned}
& x_{n+1}=\left(1-a_{n}\right) x_{n}+a_{n}\left(J_{\rho}^{\phi}\left[\eta\left(x_{n}\right)-\rho\left(\gamma_{1} T\left(y_{n}\right)+x_{n}-y_{n}\right)\right]\right), \\
& y_{n+1}=\left(1-b_{n}\right) y_{n}+b_{n}\left(J_{\rho}^{\phi}\left[\eta\left(y_{n}\right)-\rho\left(\gamma_{2} T\left(x_{n}\right)+y_{n}-x_{n}\right)\right]\right),
\end{aligned}
$$

where $0 \leq a_{n} \leq 1,0 \leq b_{n} \leq 1$.

Method 2 Let Hypothesises A and B hold. For any $\left(x_{0}, y_{0}\right) \in B \times B$, calculate $\left(x_{n+1}, y_{n+1}\right) \in B \times B$ :

$$
\begin{gathered}
x_{n+1}=\left(1-a_{n}\right) x_{n}+a_{n}\left(J_{\rho}^{\phi}\left[\eta\left(x_{n}\right)-\rho\left(\gamma_{1} T\left(y_{n}\right)+x_{n}-y_{n}\right)\right]\right), \\
y_{n+1}=\left(1-b_{n}\right) y_{n}+b_{n}\left(J_{\rho}^{\phi}\left[\eta\left(y_{n}\right)-\rho\left(\gamma_{2} T\left(x_{n+1}\right)+y_{n}-x_{n+1}\right)\right]\right),
\end{gathered}
$$

where $0 \leq a_{n} \leq 1,0 \leq b_{n} \leq 1$.

Method 3 Let Hypothesises A and B hold. For any $\left(x_{0}, y_{0}\right) \in B \times B$, calculate $\left(x_{n+1}, y_{n+1}\right) \in B \times B$ :

$$
\begin{gathered}
x_{n+1}=\left(1-a_{n}\right) x_{n}+a_{n}\left(J_{\rho}^{\phi}\left[\eta\left(x_{n}\right)-\rho\left(\gamma_{1} T\left(y_{n}\right)+x_{n}-y_{n}\right)\right]\right), \\
y_{n+1}=J_{\rho}^{\phi}\left[\eta\left(y_{n}\right)-\rho\left(\gamma_{2} T\left(x_{n}\right)+y_{n}-x_{n}\right)\right],
\end{gathered}
$$

where $0 \leq a_{n} \leq 1$.

Method 4 Let Hypothesises A and B hold. For any $\left(x_{0}, y_{0}\right) \in B \times B$, calculate $\left(x_{n+1}, y_{n+1}\right) \in B \times B$ :

$$
\begin{gathered}
x_{n+1}=\left(1-a_{n}\right) x_{n}+a_{n}\left(J_{\rho}^{\phi}\left[\eta\left(x_{n}\right)-\rho\left(\gamma_{1} T\left(y_{n}\right)+x_{n}-y_{n}\right)\right]\right), \\
y_{n+1}=J_{\rho}^{\phi}\left[\eta\left(y_{n}\right)-\rho\left(\gamma_{2} T\left(x_{n+1}\right)+y_{n}-x_{n+1}\right)\right],
\end{gathered}
$$

where $0 \leq a_{n} \leq 1$.

Remark 2 By Lemma 1, methods 1-4 are well pose.

Theorem 1 Let Hypothesises A and B hold. Let $\eta-I$ be s-Lipschitz continuous. Suppose that $\left(x^{*}, y^{*}\right)$ is a solution of SMNVI (1). Additionally, we assume that operators $\gamma_{1} T-I$ and $\gamma_{2} T-I$ are $s_{1}-$ Lipschitz and $s_{2}-$ Lipschitz continuous, respectively, such that $s_{2} b_{n}-a_{n}>0, s_{1} a_{n}-b_{n}>0$, and $1>\rho>0$ satisfies

$$
\rho<\frac{a_{n}(\alpha-s-1)}{b_{n} s_{2}-a_{n}}, \quad \rho<\frac{b_{n}(\alpha-s-1)}{a_{n} s_{1}-b_{n}} .
$$

Then the sequence $\left\{\left(x_{n}, y_{n}\right)\right\}$ generated by Method 1 converges to $\left(x^{*}, y^{*}\right)$.

Proof. Since $\left(x^{*}, y^{*}\right)$ is a solution of SMNVI (1), we have

$$
x^{*}=J_{\rho}^{\phi}\left[\eta\left(x^{*}\right)-\rho\left(\gamma_{1} T\left(y^{*}\right)+x^{*}-y^{*}\right)\right],
$$

then

$$
x^{*}=\left(1-a_{n}\right) x^{*}+a_{n}\left(J_{\rho}^{\phi}\left[\eta\left(x^{*}\right)-\rho\left(\gamma_{1} T\left(y^{*}\right)+x^{*}-y^{*}\right)\right]\right) .
$$

Since $\gamma_{1} T-I$ is $s_{1}$-Lipschitz continuous, $\eta: B \rightarrow B^{*}$ is $\alpha$-strongly monotone and $\eta-I$ is $s$-Lipschitz continuous, we have

$$
\begin{aligned}
& \left\|x_{n+1}-x^{*}\right\| \\
= & \left\|\left(1-a_{n}\right) x_{n}+a_{n}\left(J_{\rho}^{\phi}\left[\eta\left(x_{n}\right)-\rho\left(\gamma_{1} T\left(y_{n}\right)+x_{n}-y_{n}\right)\right]\right)-\left(1-a_{n}\right) x^{*}-a_{n}\left(J_{\rho}^{\phi}\left[\eta\left(x^{*}\right)-\rho\left(\gamma_{1} T\left(y^{*}\right)+x^{*}-y^{*}\right)\right]\right)\right\| \\
\leq & \left(1-a_{n}\right)\left\|x_{n}-x^{*}\right\|+a_{n}\left\|J_{\rho}^{\phi}\left[\eta\left(x_{n}\right)-\rho\left(\gamma_{1} T\left(y_{n}\right)+x_{n}-y_{n}\right)\right]-J_{\rho}^{\phi}\left[\eta\left(x^{*}\right)-\rho\left(\gamma_{1} T\left(y^{*}\right)+x^{*}-y^{*}\right)\right]\right\| \\
= & \left(1-a_{n}\right)\left\|x_{n}-x^{*}\right\|+a_{n} \| J_{\rho}^{\phi}\left[\eta\left(x_{n}\right)-\rho\left(\gamma_{1} T\left(y_{n}\right)+x_{n}-y_{n}\right)\right]-J_{\rho}^{\phi}\left[\eta\left(x_{n}\right)-\rho\left(\gamma_{1} T\left(y^{*}\right)+x_{n}-y^{*}\right)\right]+ \\
& J_{\rho}^{\phi}\left[\eta\left(x_{n}\right)-\rho\left(\gamma_{1} T\left(y^{*}\right)+x_{n}-y^{*}\right)\right]-J_{\rho}^{\phi}\left[\eta\left(x^{*}\right)-\rho\left(\gamma_{1} T\left(y^{*}\right)+x^{*}-y^{*}\right)\right] \| \\
\leq & \left(1-a_{n}\right)\left\|x_{n}-x^{*}\right\|+\frac{a_{n}}{\alpha}\left\|\rho\left(\gamma_{1} T\left(y_{n}\right)-y_{n}-\gamma_{1} T\left(y^{*}\right)+y^{*}\right)\right\|+\frac{a_{n}}{\alpha}\left\|\eta\left(x_{n}\right)-x_{n}+(1-\rho) x_{n}-\eta\left(x^{*}\right)+x^{*}+(\rho-1) x^{*}\right\| \\
\leq & \left(1-a_{n}\right)\left\|x_{n}-x^{*}\right\|+\frac{\rho a_{n} s_{1}}{\alpha}\left\|y_{n}-y^{*}\right\|+\frac{a_{n}(s+1-\rho)}{\alpha}\left\|x_{n}-x^{*}\right\|,
\end{aligned}
$$


where the second inequality obtained by Lemma 2 .

Analogously for $y^{*}$, we obtain

$$
\left\|y_{n+1}-y^{*}\right\| \leq\left(1-b_{n}\right)\left\|y_{n}-y^{*}\right\|+\frac{\rho b_{n} s_{2}}{\alpha}\left\|x_{n}-x^{*}\right\|+\frac{b_{n}(s+1-\rho)}{\alpha}\left\|y_{n}-y^{*}\right\| .
$$

Hence, we have

$$
\left\|x_{n+1}-x^{*}\right\|+\left\|y_{n+1}-y^{*}\right\| \leq \max \left\{\theta_{1}, \theta_{2}\right\}\left(\left\|x_{n}-x^{*}\right\|+\left\|y_{n}-y^{*}\right\|\right),
$$

where, $\theta_{1}=1-a_{n}+\frac{a_{n}(s+1-\rho)+\rho b_{n} s_{2}}{\alpha}, \theta_{2}=1-b_{n}+\frac{\rho a_{n} s_{1}+b_{n}(s+1-\rho)}{\alpha}$. Define the norm $\|\cdot\|_{*}$ on $B \times B$ as:

$$
\|(u, v)\|_{*}=\|u\|+\|v\|, \quad \forall(u, v) \in B \times B .
$$

Obviously, $\left(B \times B,\|\cdot\|_{*}\right)$ is a Banach space. Hence, (6) implies

$$
\left\|\left(x_{n+1}, y_{n+1}\right)-\left(x^{*}, y^{*}\right)\right\|_{*} \leq \max \left\{\theta_{1}, \theta_{2}\right\}\left\|\left(x_{n}, y_{n}\right)-\left(x^{*}, y^{*}\right)\right\|_{*} .
$$

By (5), we have $\theta_{1}, \theta_{2} \in(0,1)$. Hence, by Lemma 4 , we have

$$
\left(x_{n}, y_{n}\right) \rightarrow\left(x^{*}, y^{*}\right), \quad n \rightarrow \infty
$$

which completes the proof.

Similarly to the proof of theorem 1, we have the following theorems.

Theorem 2 Let Hypothesises A and B hold. Let $\eta$ - I be s-Lipschitz continuous. Suppose that $\left(x^{*}, y^{*}\right)$ is a solution of SMNVI (1). Additionally, we assume that operators $\gamma_{1} T-I$ and $\gamma_{2} T-I$ are $s_{1}-$ Lipschitz and $s_{2}-$ Lipschitz continuous, respectively, such that $s_{2} b_{n}-a_{n}>0, s_{1} a_{n}-b_{n}>0$, and $1>\rho>0$ satisfies

$$
\begin{gathered}
b_{n} s_{2} a_{n} s_{1} \rho^{2}+\left(\alpha a_{n} s_{1}-\alpha b_{n}\right) \rho-\alpha^{2} b_{n}<0, \\
-a_{n} b_{n} s_{2} \rho^{2}+\hat{b} \rho-\alpha^{2}<0,
\end{gathered}
$$

where

$$
\hat{b}=\alpha b_{n} s_{2}-\alpha a_{n}-\alpha a_{n} b_{n} s_{2}+a_{n} s b_{n} s_{2}+a_{n} b_{n} s_{2} .
$$

Then the sequence $\left\{\left(x_{n}, y_{n}\right)\right\}$ generated by Method 2 converges to $\left(x^{*}, y^{*}\right)$.

Theorem 3 Let Hypothesises A and B hold. Let $\eta-I$ be $s$-Lipschitz continuous. Suppose that $\left(x^{*}, y^{*}\right)$ is a solution of SMNVI (1). Additionally, we assume that operators $\gamma_{1} T-I$ and $\gamma_{2} T-I$ are $s_{1}-$ Lipschitz and $s_{2}-$ Lipschitz continuous, respectively, such that $s_{2}-a_{n}>0, s_{1} a_{n}-1>0,1>\rho>0$ satisfies

$$
\rho<\frac{a_{n}(\alpha-s-1)}{s_{2}-a_{n}}, \quad \rho<\frac{\alpha-1-s}{s_{1} a_{n}-1}
$$

Then the sequence $\left\{\left(x_{n}, y_{n}\right)\right\}$ generated by Method 3 converges to $\left(x^{*}, y^{*}\right)$.

Theorem 4 Let Hypothesises $A$ and B hold. Let $\eta-I$ be $s$-Lipschitz continuous. Suppose that $\left(x^{*}, y^{*}\right)$ is a solution of SMNVI (1). Additionally, we assume that operators $\gamma_{1} T-I$ and $\gamma_{2} T-I$ are $s_{1}-$ Lipschitz and s $s_{2}-$ Lipschitz continuous, respectively, such that $1>\rho>0$ satisfies

$$
\begin{gathered}
s^{2} \rho+\left(\alpha-a_{n} s_{2} s-a_{n} s_{2}-\alpha s_{2}+\alpha a_{n} s_{2}\right) \rho+a_{n} \alpha^{2}-a_{n} \alpha s+a_{n} \alpha>0, \\
a_{n} s_{1} s_{2} \rho^{2}+\left(a_{n} s_{1} \alpha-\alpha\right) \rho+\alpha s+\alpha-\alpha^{2}<0
\end{gathered}
$$

Then the sequence $\left\{\left(x_{n}, y_{n}\right)\right\}$ generated by Method 4 converges to $\left(x^{*}, y^{*}\right)$.

\section{Acknowledgements}

The work was supported by Natural Science Foundation of Guangdong Province, China (Grant No. S2012040007993) and Educational Commission of Guangdong Province, China (Grant No. 2012LYM_0122). 


\section{References}

Duvaut, G., \& Lions, J. L. (1976). Inequalities in Mechanics and Physics. Berlin: Springer-Verlag. http://dx.doi.org/10.1007/978-3-642-66165-5

Facchinei, F., \& Pang, J. S. (2003). Finite-Dimensional Variational Inequalities and Complementarity Problems. New York: Springer.

Ferris, M., \& Pang, J. S. (1997). Engineering and economics applications of complementarity problems. SIAM Review, 39, 669-713. http://dx.doi.org/10.1137/S0036144595285963

Huang, Z. Y., \& Noor, M. A. (2007). An explicit projection method for a system of nonlinear variational inequalities with different $(\gamma, r)$-cocoercive mappings. Applied Mathematics and Computation, 190, 356-361. http://dx.doi.org/10.1016/j.amc.2007.01.032

Kazmi, K. R., \& Khan, F. A. (2007). Auxiliary problems and algorithm for a system of generalized variational-like inequality problems. Applied Mathematics and Computation, 187, 789-796. http://dx.doi.org/10.1016/j.amc.2006.08.157

Verma, R. U. (2001a). A system of generalized auxiliary problems principle and a system of variational inequalities. Mathematical Inequalities and Applications, 4, 443-453. http://dx.doi.org/10.7153/mia-04-39

Verma, R. U. (2001b). Projection methods, algorithms, and a new system of nonlinear variational inequalities.

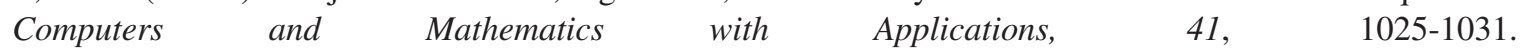
http://dx.doi.org/10.1016/S0898-1221(00)00336-9

Xia, F. Q., \& Huang, N. J. (2008). Algorithm for solving a new class of general mixed variational inequalities in Banach spaces. Journal of Computational and Applied Mathematics, 220, 632-642. http://dx.doi.org/10.1016/j.cam.2007.09.011

\section{Copyrights}

Copyright for this article is retained by the author(s), with first publication rights granted to the journal.

This is an open-access article distributed under the terms and conditions of the Creative Commons Attribution license (http://creativecommons.org/licenses/by/3.0/). 